\title{
Erratum to: Synthesis, X-ray structure and theoretical study of benzazole thioether and its zinc complex as corrosion inhibitors for steel in acidic medium
}

\author{
Bilel Mezhoud ${ }^{1,2} \cdot$ Mehdi Bouchouit $^{1}$. \\ Elhadi Mohamed Said ${ }^{1} \cdot$ Lyamine Messaadia $^{3}$. \\ Ali Belfaitah ${ }^{4}$ - Hocine Merazig ${ }^{1}$ Aissa Chibani ${ }^{1}$. \\ Sofiane Bouacida ${ }^{1,5} \cdot$ Abdelmalek Bouraiou ${ }^{1}$ \\ Published online: 17 November 2016 \\ (C) Springer Science+Business Media Dordrecht 2016
}

\section{Erratum to: Res Chem Intermed (2016) 42:7447-7470 DOI 10.1007/s11164-016-2546-3}

In the original publication of the article, there exists an inversion between the family name and the first name of all authors. The correct version is given in this erratum:

B. Mezhoud, M. Bouchouit, E. M. Said, L. Messaadia, A. Belfaitah, H. Merazig, A. Chibani, S. Bouacida, A. Bouraiou

The online version of the original article can be found under doi:10.1007/s11164-016-2546-3.

$\triangle$ Abdelmalek Bouraiou

bouraiou.abdelmalek@yahoo.fr

1 Unité de Recherche de CHimie de l'Environnement, et Moléculaire Structurale, CHEMS, Université des frères Mentouri, Constantine, Algeria

2 Centre de Recherche Scientifique et Technique en Analyses Physico-chimiques (CRAPC), Plateau Technique d'Analyse Physico-Chimiques, Université des frères Mentouri, Constantine, Algeria

3 Laboratoire Energétique Appliquée et Matériaux, Université de Jijel, 18000 Jijel, Algeria

4 Laboratoire des Produits Naturels d'Origine Végétale et de Synthèse Organique, Université des frères Mentouri, Constantine, Algeria

5 Département Sciences de la Matière, Université Oum El Bouaghi, 04000 Oum El Bouaghi, Algeria 